\title{
Pathophysiological and Psychosocial Features in Functional Vomiting
}

TO THE EDITOR: I read the report on pathophysiological and psychosocial aspect of functional vomiting (FV) by Zhao et $\mathrm{al}^{1}$ with a great interest. The authors suggested that patients with functional vomiting have abnormal psychological status, gastric motility and hypersensitivity compared to healthy subjects. FV is defined as recurrent vomiting in the absence of all known medical and psychiatric causes. ${ }^{2}$ The differential diagnosis of vomiting is extensive and FV should be distinguished from vomiting that occurs occasionally in patients with functional dyspepsia (FD), especially dysmotility-like dyspepsia. In addition, FV group in this study have many overlaps (84.2\%) with FD. So, results in their study are not only specific for FV but also FD. The use of electrogastrography (EGG) is not widely accepted, although dysarhythmias may be recorded in some patient unexplained nausea and vomiting with normal gastric emptying. ${ }^{3}$ In selected patients, EGG may be complementary to gastric emptying study. Therefore, gastric motility evaluation such as gastric emptying scintiography can be essential to exclude unexplained vomiting. In EGG finding, the authors described FV patients has $50 \%$ with abnormal power ratio. However, I wonder what "abnormal" means and its clinical implication.

Psychosocial factors are widely believed to be prominent features of functional gastrointestinal disorders. ${ }^{4}$ In this study, psychological state (anxiety and depression), social stress and neuroticism are associated with $\mathrm{FV}$. These factors are also related with abnormal gastric sensorimotor function by perfusion nutrition load test (P-NLT). Although the author presented the relationship between psychosocial factors and the amount of overlaps or gastric sensorimotor function in Tables 4 and 6, the rela- tionship between psychosocial factors and symptoms of $\mathrm{FV}$, which is main focus of this study, could be also presented. In addition, as mentioned in discussion, P-NLT is not widely accepted tool for assessment of gastric accommodation and sensation. Thus, the use of electric barostat could strengthen the result of this study.

Despite several limitations of the present study, it gives us more insight into the pathophysiology of FV. Further studies with large cases and other sensorimotor function test are needed to reinforce the findings in this study.

\section{Jung Mook Kang, MD \\ Department of Internal Medicine Seoul National University College of Medicine Seoul, Korea}

1. Zhao Y, Ke M, Wang Z, et al. Pathophysiological and psychosocial study in patients with functional vomiting. J Neurogastroenterol Motil 2010;16:274-280.

2. Tack J, Talley NJ, Camilleri M, et al. Functional gastroduodenal disorders. Gastroenterology 2006;130:1466-1479.

3. Parkman HP, Hasler WL, Barnett JL, Eaker EY; American Motility Society Clinical GI Motility Testing Task Force. Electrogastrography: a document prepared by the gastric section of the American Motility Society Clinical GI Motility Testing Task Force. Neurogastroenterol Motil 2003;15:89-102.

4. Levy RL, Olden KW, Naliboff BD, et al. Psychosocial aspects of the functional gastrointestinal disorders. Gastroenterology 2006;130:14471458.

Conflicts of interest: None. 